Tropical Journal of Pharmaceutical Research July 2020; 19 (7): 1441-1448

ISSN: $1596-5996$ (print); 1596-9827 (electronic)

(C) Pharmacotherapy Group, Faculty of Pharmacy, University of Benin, Benin City, 300001 Nigeria.

\title{
Comparative studies of the anti-thrombotic effects of saffron and HongHua based on network pharmacology
}

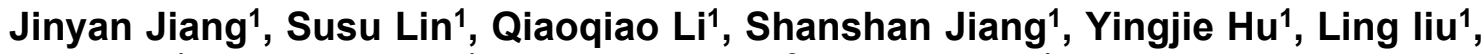 \\ Yu Jiang ${ }^{1}$, Shuting Chen ${ }^{1}$, Yingpeng Tong ${ }^{2 *}$, Ping Wang ${ }^{1 *}$ \\ ${ }^{1}$ College of Pharmaceutical Science, Zhejiang University of Technology, Hangzhou, 310014, ${ }^{2}$ Institute of Natural Medicine and \\ Health Product, School of Advanced Study, Taizhou University, Taizhou, China, 318000
}

*For correspondence: Email: wangping45@zjut.edu.cn; Tel: +86-13634108877

\begin{abstract}
Purpose: To investigate the comparative anti-thrombotic effects of saffron and Honghua, and also to explore possible mechanisms in thrombosis based on network pharmacology.

Methods: A network pharmacology model was used for bioactive components, targets and pathways for saffron and HongHua via Traditional Chinese Medicine Systems Pharmacology Database and Analysis Platform (TCMSP), PharmMapper, Genecard, Uniprot and KEGG databases. In animal experiments, 72 rats were randomly divided into 9 groups: normal control group (NC), model control group (MC), crocetin groups (80, 40, $20 \mathrm{mg} / \mathrm{kg}$ ), hydroxysafflor yellow A(HSYA) groups (80, 40, 20 $\mathrm{mg} / \mathrm{kg}$ ), and aspirin group (40 mg/kg). Using in vitro thrombosis models and an acute blood stasis model in vivo, the anti-thrombotic effects of these treatments on clotting time, hemorheology parameters, Thromboxane B2 (TXB2), plasmin activator inhibitor (PAI), protein C (PC), protein S (PS), and thrombinantithrombin complex (TAT) were determined and comparisons made for saffron and HongHua. Results: Five potential compounds, 16 anti-thrombotic targets and 27 pathways were predicted for saffron, while 22 compounds, 37 disease targets and 35 pathways were found for HongHua $(p<0.05)$. Pharmacological experiments revealed that crocetin and HSYA had significant effects on thrombus length, thrombus wet/dry mass, whole blood viscosity (WBV), erythrocyte aggregation index (EAI), clotting time and D-dimer for the high and middle groups. Unlike HSYA, crocetin also had significant and dose-dependent effects on PAI, prothrombin fragment $1+2(F 1+2)$ and $P S$ and had highly significant effects on TXB2 and TAT.

Conclusion: This research provides a systematic, comprehensive and comparative analysis of component, target and anti-thrombotic pathways of saffron and HongHua based on network pharmacology, and also shows that saffron has more significant anti-thrombotic effect than HongHua.
\end{abstract}

Keywords: Saffron; HongHua; Network pharmacology; Anti-thrombosis; Network model

\begin{abstract}
This is an Open Access article that uses a fund-ing model which does not charge readers or their institutions for access and distributed under the terms of the Creative Commons Attribution License (http://creativecommons.org/licenses/by/4.0) and the Budapest Open Access Initiative (http://www.budapestopenaccessinitiative.org/read), which permit unrestricted use, distribution, and
\end{abstract} reproduction in any medium, provided the original work is properly credited.

Tropical Journal of Pharmaceutical Research is indexed by Science Citation Index (SciSearch), Scopus, International Pharmaceutical Abstract, Chemical Abstracts, Embase, Index Copernicus, EBSCO, African Index Medicus, JournalSeek, Journal Citation Reports/Science Edition, Directory of Open Access Journals (DOAJ), African Journal Online, Bioline International, Open-J-Gate and Pharmacy Abstracts

\section{INTRODUCTION}

Saffron (Crocus sativus L.) is native to Iran and Spain. The main bioactive compounds in saffron are terpenoids, flavonoids, anthraquinones and cyclohexene [1]. Saffron was first recorded in "Ben cao pin hui jing yao" in China. HongHua (Carthamus tinctorius L.), which originated from 
China, is rich in flavonoids, pigments, phenolic acids and alkaloids. As representative TCM for cardiovascular diseases, saffron and HongHua are associated with long medicinal histories and rich clinical values [3,4]. Indeed, in many "Chinese Pharmacy" textbooks, saffron is considered an "additional medicine" of HongHua. However, chemical studies have revealed only few identical components $[2,5]$. Therefore, there is need for comparative studies on their effects on "blood stasis".

Thrombosis is implicated in the etiology of cardiovascular and cerebrovascular diseases [6]. Statistics indicate that thrombosis, with a global annual death of 12 million, has become one of the biggest killer diseases [7]. In the view of Chinese medicine, modern thrombosis belongs to "blood stasis", which has been already confirmed in "Fifty-two Patient Prescriptions", and modern research also renamed it blood stasis syndrome [8]. However, the single component targets of modern clinical treatment tend to mask the multi target advantages of TCM, thereby limiting the maximization of the efficacy of TCM. However, the application of network pharmacology will help to achieve therapeutic effects of TCM more accurately.

Thus, this study was performed to predict the possible anti-thrombotic effect of saffron and HongHua based on network pharmacology using their representative compounds (crocetin and HSYA).

\section{METHODS}

\section{Collection and screening of compound}

Based on TCMSP, "saffron" and "HongHua" were imported as key words for retrieval. Their potential bioactive components were collected under the screening conditions of oral bioavailability $(\mathrm{OB})>30 \%$ and drug-likeness $(\mathrm{DL})>0.18$.

\section{Target prediction for compound and disease}

The bioactive components obtained from TCMSP were searched in PharmMapper, Drugbank and Genecards databases to obtain potential targets. The disease targets were searched using the Genecards database, and the corresponding thrombotic disease targets were screened.

\section{GO and KEGG analysis of pathways}

The protein targets and gene names collected in 2.2 were summarized and imported into the DAVID database to analyze the signal pathways through enrichment of Gene Ontology (GO) and KEGG Pathways.

\section{Construction and analysis of the network model}

The top 20 important signal pathways that were identified in the DAVID database were selected for analysis. Then, the bioactive components, targets and pathways of saffron and HongHua were sorted out. The component-target and component-target-pathway interactions of saffron and HongHua were constructed. Finally, the results were introduced into the database using Cytoscape 3.6.0. and network models were constructed to analyze the interactions. Finally, effective targets and pathways were predicted according to degree values. The larger the degree values, the closer the interaction with anti-thrombosis, and the greater the effect on thrombosis.

\section{Experimental validation}

\section{Chemicals and reagents}

Crocetin was self-produced in the laboratory (purity > 98 \%), HSYA (CAS: 78281-02-4, LOT: R25A9F59977; purity $\geqq 98 \%$ ) was purchased from Shanghai Yuanye Biotechnology Co. Ltd. LEpinephrine hydrochloride (CAS: 55-31-2, LOT: Y03J7C15662, HPLC $\geqq 99 \%$ ) was purchased from Shanghai Yuanye Biotechnology Co. Ltd. Aspirin enteric-coated tablets were obtained from Chenxin Pharmaceutical Co. Ltd.

\section{Animals and drug administration}

Seventy-two adult male Sprague-Dawley rats (240 - $260 \mathrm{~g})$ were purchased from Zhaoyan (Suzhou) New Pharmaceutical Research Center Co. Ltd. (Certificate of Qualification: no. 201920610) were placed in a room with a 12-h light/12-h dark cycle, and provided sufficient food and water. The animal study was approved by the Animal Ethics Committee of the Animal Center of Zhejiang University of Technology (approval no. 20191018090). The study was conducted in accordance with the guidelines for the Care and Use of Laboratory Animals [9]. The rats were randomly divided into 9 groups: normal control group (NC), model control group (MC), crocetin groups $(80,40,20 \mathrm{mg} / \mathrm{kg})$, HSYA groups $(80,40,20 \mathrm{mg} / \mathrm{kg})$, and aspirin group (40 mg/kg).

\section{Acute blood stasis model in vivo}

All treatments were administered via oral administration once daily for 8 days. NC and MC groups were administered $0.9 \%(w / v) \quad \mathrm{NaCl}$. 
During the last two days, rats in each group except the NC group were injected subcutaneously with adrenaline hydrochloride (Adr) at a dose of $0.8 \mathrm{mg} / \mathrm{kg}$ ) twice at intervals of $4 \mathrm{~h}$, and placed in ice-cold water $\left(0-2{ }^{\circ} \mathrm{C}\right)$ for 5 min in-between injections. Finally, the rats were fasted overnight and anesthetized with chloral hydrate $(350 \mathrm{mg} / \mathrm{kg})$. Blood samples were collected on the following day.

\section{In vitro thrombosis model}

The rat groups were same as previously noted. One hour after the last administration, $1.8 \mathrm{ml}$ blood was taken from the retro-orbital plexus and quickly injected into a thrombus ring which was spun at $37{ }^{\circ} \mathrm{C}$ at $16 \mathrm{rpm}$ for $16 \mathrm{~min}$. Finally, the thrombus length and wet mass were obtained, and the thrombus was dried at $60{ }^{\circ} \mathrm{C}$ for $2 \mathrm{~h}$ in an oven to obtain the dry mass.

\section{Assessment of clotting time}

As described in section 2.5.3.1, one hour after administration on the 7th day, a cut was made 5 $\mathrm{mm}$ away from the tail of each rat, and blood was allowed to drop on a clean glass slide. The blood drop diameter was maintained at $5-10 \mathrm{~mm}$. The clotting time of the blood was determined in 10second intervals [10].

\section{Determination of hemorheology parameters}

Blood obtained in heparin sodium anticoagulated vials was applied for determination of whole blood viscosity (WBV) with three shear rates (150/sec, 60/sec and 10/sec). Hematocrit and erythrocyte aggregation index (EAI) were assessed within 1 hour using an automatic blood rheometer (LBY - N7500B, Beijing Pulisheng Instrument Co. Ltd.) and an instrument that measures automatic erythrocyte settling rate (LBY - XC40, Beijing Pulisheng Instrument Co. Ltd.).

\section{Determination of coagulation indices}

The coagulation parameters TXB2, PAI, 6-ketoPGFa, PC, PS, F1+2, TAT and D-dimer were measured using ELISA kits (Shanghai, China) following the instructions provided in the kit manual.

\section{Statistical analysis}

The data are presented as mean \pm standard deviation (SD). Student's $t$-test was used to compare mean values between groups with the aid of SPSS software. Values of $p<0.05$ were considered statistically significant.

\section{RESULTS}

\section{Profile of bioactive compounds in saffron and HongHua}

A total of 70 saffron and 189 HongHua compounds were obtained from the TCMSP platform. However, only 5 potential saffron compounds and 22 HongHua compounds were obtained after screening. These results are shown in Table 1.

Target analysis of saffron and HongHua in disease

Potential protein targets were assessed [11]. A total of 272 protein targets of saffron and 449 protein targets of HongHua were obtained, while 749 protein targets of thrombus were identified. After screening and exclusion, 16 anti-thrombotic targets of saffron and 37 anti-thrombotic targets of HongHua were identified. The overlap analysis of thrombotic targets showed that 16 targets of saffron were completely included in HongHua, indicating that the 16 targets were common gene targets of saffron, HongHua and thrombus.

\section{GO and KEGG analysis of pathway}

DAVID analysis revealed 27 signal pathways in saffron and 35 in HongHua $(p<0.05)$ [12], and 23 of these pathways were identical. GO analysis results revealed 82 terms in saffron, including 55 biological process (BP) terms, 12 cellular component (CC) terms and 15 molecular function (MF) terms. Among these, blood coagulation, extracellular space and serine endopeptidase activity were the most significant terms for thrombosis treatment. There was a total of 138 terms in HongHua, including $99 \mathrm{BP}$ terms, $19 \mathrm{CC}$ terms and $20 \mathrm{MF}$ terms. The most enriched pathways of BP and $\mathrm{CC}$ in HongHua were the same in saffron. With respect to molecular function, the extracellular region was regarded as the most important thrombotic pathway in HongHua. These results are shown in Figures 1, 2 and 3.

\section{Construction and analysis of the network model}

After classification and summarizing, the interaction between saffron and HongHua was established. The network model of saffron and HongHua was obtained using Cytoscape 3.6.0 software, as shown in Figure 4. 
Table 1: Basic information for 27 active compounds in saffron and HongHua

\begin{tabular}{|c|c|c|c|c|c|}
\hline Medicine & Code & Compound & OB\% & $\mathrm{DL}$ & Molecular weight \\
\hline \multirow[t]{5}{*}{ Saffron } & $\mathrm{XHH1}$ & N-heptanal & 79.74 & 0.59 & 352.42 \\
\hline & $\mathrm{XHH} 2$ & Isorhamnetin & 49.6 & 0.31 & 316.28 \\
\hline & $\mathrm{XHH} 3$ & Quercetin & 46.43 & 0.28 & 302.25 \\
\hline & $\mathrm{XHH} 4$ & Crocetin & 35.3 & 0.26 & 328.44 \\
\hline & $\mathrm{XHH} 5$ & Kaempferol & 41.88 & 0.24 & 286.25 \\
\hline \multirow[t]{23}{*}{ HongHua } & $\mathrm{HH} 1$ & Stigmasterol & 43.83 & 0.76 & 412.77 \\
\hline & $\mathrm{HH} 2$ & Baicalin & 40.12 & 0.75 & 446.39 \\
\hline & $\mathrm{HH} 3$ & Beta-sitosterol & 36.91 & 0.75 & 414.79 \\
\hline & $\mathrm{HH} 4$ & Poriferast-5-en-3beta-ol & 36.91 & 0.75 & 414.79 \\
\hline & HH5 & CLR & 37.87 & 0.68 & 386.73 \\
\hline & $\mathrm{HH} 6$ & Lignan & 43.32 & 0.65 & 458.55 \\
\hline & $\mathrm{HH} 7$ & Beta-carotene & 37.18 & 0.58 & 536.96 \\
\hline & $\mathrm{HH} 8$ & Flavoxanthin & 60.41 & 0.56 & 584.96 \\
\hline & $\mathrm{HH} 9$ & Phytoene & 39.56 & 0.5 & 545.04 \\
\hline & $\mathrm{HH} 10$ & Phytofluene & 43.18 & 0.5 & 543.02 \\
\hline & $\mathrm{HH} 11$ & 4-[(E)-4-(3,5-dimethoxy-4-oxo-1- & & & \\
\hline & & $\begin{array}{l}\text { cyClohexa-2,5-dienylidene)but-2- } \\
\text { enylidene]-2,6-dimethoxycyclohexa- } \\
\text { 2,5-dien-1-one }\end{array}$ & 48.47 & 0.36 & 356.40 \\
\hline & $\mathrm{HH} 12$ & Pyrethrin II & 48.36 & 0.35 & 372.50 \\
\hline & $\mathrm{HH} 13$ & Lupeol-palmitate & 33.98 & 0.32 & 665.26 \\
\hline & $\mathrm{HH} 14$ & Quercetagetin & 45.01 & 0.31 & 318.25 \\
\hline & $\mathrm{HH} 15$ & Quercetin & 46.43 & 0.28 & 302.25 \\
\hline & $\mathrm{HH} 16$ & 6-Hydroxykaempferol & 62.13 & 0.27 & 302.25 \\
\hline & $\mathrm{HH} 17$ & Luteolin & 36.16 & 0.25 & 286.25 \\
\hline & $\mathrm{HH} 18$ & 6-Hydroxynaringenin & 33.23 & 0.24 & 288.27 \\
\hline & $\mathrm{HH} 19$ & Kaempferol & 41.88 & 0.24 & 286.25 \\
\hline & $\mathrm{HH} 20$ & Baicalein & 33.52 & 0.21 & 270.25 \\
\hline & $\mathrm{HH} 21$ & qt_Carthamone & 51.03 & 0.2 & 286.25 \\
\hline & $\mathrm{HH} 22$ & $\begin{array}{c}\text { 7,8-Dimethyl-1H-pyrimido } \\
{[5,6-\text { g]Quinoxaline-2,4-dione }}\end{array}$ & 45.75 & 0.19 & 242.26 \\
\hline
\end{tabular}

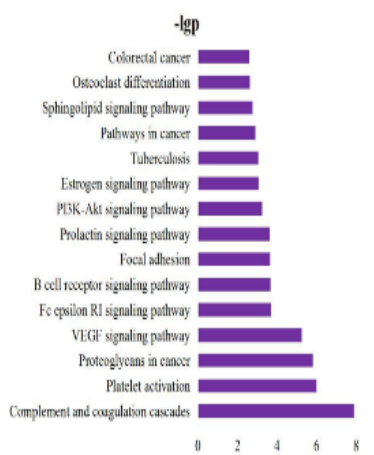

(A)

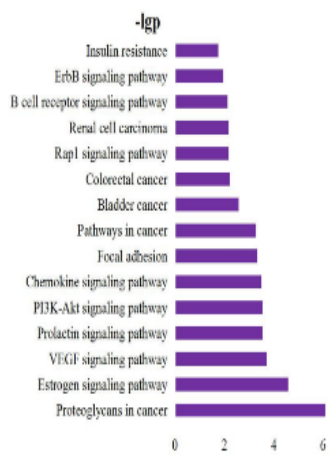

(B)

Figure 1: KEGG pathway enrichment of HongHua (A) and saffron $(B)$ against thrombus

The results revealed 41 nodes and 165 edges in the saffron network diagram and 71 nodes and 450 edges in the HongHua network diagram. Thus, saffron had fewer compounds and network edges than HongHua. In addition, degree values were used to predict function effect on thrombus. The degree values for quercetin and kaempferol were the highest for saffron, while AKT1 and RAC1 were the most related targets. With respect to HongHua, 6-hydroxy-kaempferol and quercetin were the most related potential

compounds, and only MAPK8 did not appear in the top 5 genes for HongHua.

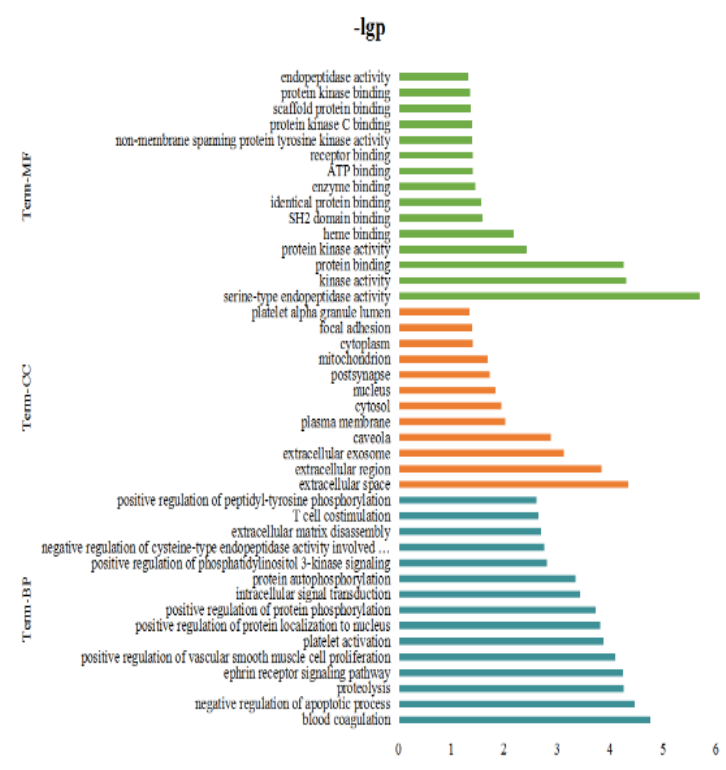

Figure 2: Gene Ontology terms of predicted target of saffron against thrombus 


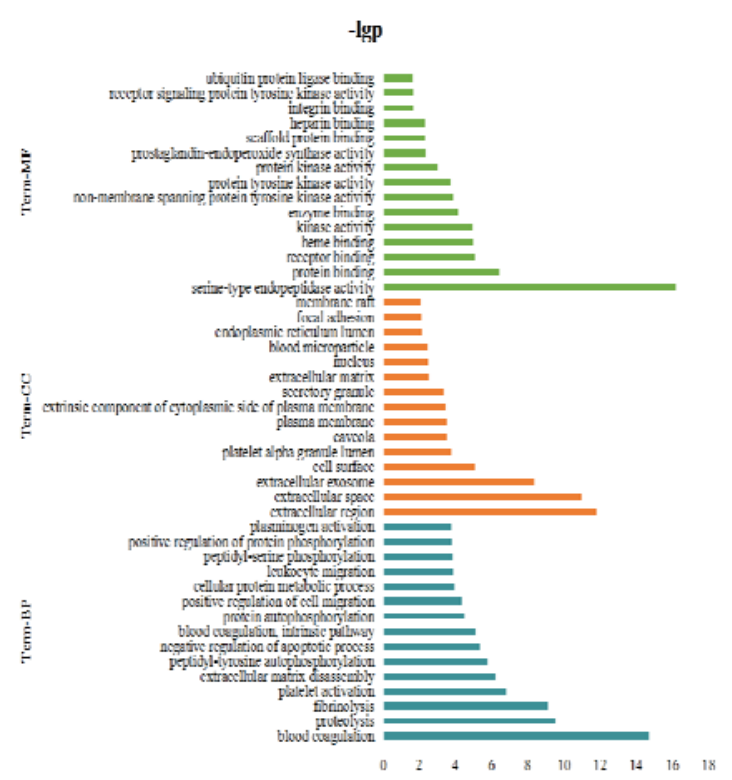

Figure 3: Gene Ontology terms of the predicted target of HongHua against thrombus

Thus, it was assumed to be a pivotal target of action for the two compounds. Moreover, many potential targets and signaling pathways were similar in the network interaction. Among the top 10 degree values, there were 7 common potential targets, i.e., AKT1, RAC1, SRC, GSK3beta, NOS3, HGF and MMP9, and 7 pathways including cancer pathway, VEGF signal pathway, platelet activation, PI3K-Akt signaling pathway, proteoglycan in cancer, sphingolipid signaling pathway, estrogen signaling pathway and adhesion.

\section{Determination of thrombus length, wet mass and dry mass}

Data on thrombus length and wet/dry mass are shown in Table 2. Significant changes were noted in all treatment groups compared with the MC group, indicating that crocetin and HSYA dose-dependently reduce thrombosis.

\section{Determination of hemorheology parameters}

As shown in Table 3, Crocetin and HSYA groups with high and middle doses exhibited significant treatment effects. WBV (150/S, $60 / S, 10 / S)$, Hematocrit and EAI decreased after administration, and the decrease in WBV reduced thrombus formation. The $\mathrm{H}$-crocetin group had more significant effect than the aspirin group, and differences were noted in all groups with $P<0.01$. Meanwhile, all crocetin treatment groups exhibited dose-dependent effects.

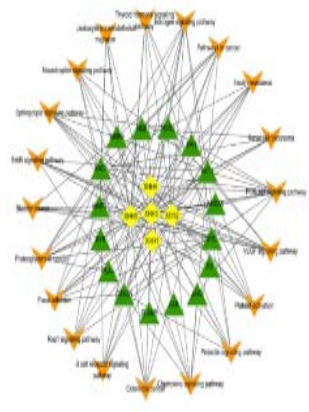

(A)

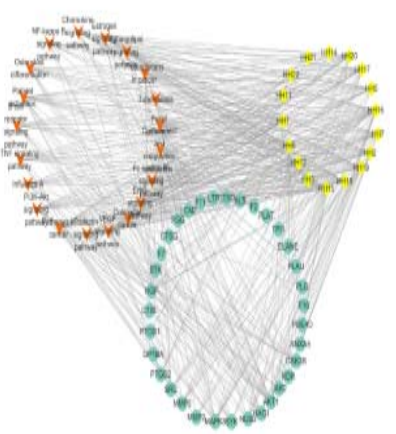

(B)
Figure 4: Network model of "compound-targetpathway" for saffron (A) and HongHua (B) against thrombus (green indicates the target, yellow indicates compound, and orange indicates pathway)

\section{Determination in ELISA analysis and clotting time}

Studies showed that thrombosis is closely related to blood coagulation and the fibrinolytic system, and the determination of relevant factors would be helpful for the evaluation of its effect. Therefore, clotting time, F1 + 2, TAT, PS, 6-ketoPGF1a, PAI, PC, TXB2 and D-dimers were determined to verify the therapeutic effect of treatment groups.

Table 2: Determination of thrombus length and wet/dry mass in rats (mean $\pm S D, n=8$ )

\begin{tabular}{|c|c|c|c|c|}
\hline Group & Dose $(\mathrm{mg} / \mathrm{kg})$ & Thrombosis length/cm & Thrombosis wet mass/g & Thrombus dry mass/g \\
\hline $\mathrm{MC}$ & 1 & $9.450 \pm 1.144$ & $0.629 \pm 0.056$ & $0.154 \pm 0.009$ \\
\hline H-Crocetin & 80 & $5.238 \pm 1.189^{\star \star *}$ & $0.413 \pm 0.066^{\star \star \star}$ & $0.139 \pm 0.009^{\star *}$ \\
\hline M-Crocetin & 40 & $6.775 \pm 1.943^{* *}$ & $0.500 \pm 0.074^{\star \star}$ & $0.143 \pm 0.004^{* *}$ \\
\hline L-Crocetin & 20 & $7.600 \pm 1.478^{* *}$ & $0.534 \pm 0.047^{* *}$ & $0.146+0.006^{*}$ \\
\hline H-HSYA & 80 & $5.363 \pm 1.205^{\star * *}$ & $0.473 \pm 0.048^{* * *}$ & $0.138 \pm 0.005^{\star * *}$ \\
\hline M-HSYA & 40 & $6.329 \pm 1.650^{\star *}$ & $0.496 \pm 0.033^{* * *}$ & $0.145 \pm 0.004^{*}$ \\
\hline L-HSYA & 20 & $6.675 \pm 1.937^{\star *}$ & $0.514 \pm 0.078^{* *}$ & $0.147 \pm 0.005^{\star}$ \\
\hline Aspirin & 50 & $6.463 \pm 1.355^{\star * \star}$ & $0.556 \pm 0.064^{*}$ & $0.145 \pm 0.005^{*}$ \\
\hline
\end{tabular}

${ }^{\star} P<0.05,{ }^{\star \star} p<0.01,{ }^{\star \star \star} p<0.001$ compared with model control group 
Table 3: Determination of hemorheology parameters in rats (mean $\pm S D, n=8$ )

\begin{tabular}{|c|c|c|c|c|c|c|}
\hline \multirow{2}{*}{ Group } & \multirow{2}{*}{ Dose $(\mathrm{mg} / \mathrm{kg})$} & \multicolumn{3}{|c|}{ WBV } & \multirow{2}{*}{ Hematocrit } & \multirow{2}{*}{ EAl } \\
\hline & & $150 / S$ & $60 / S$ & $10 / S$ & & \\
\hline $\mathrm{NC}$ & I & $6.3 \pm 0.7$ & $9.6 \pm 1.3$ & $21.1 \pm 4.5$ & $59.5 \pm 3.2$ & $3.3 \pm 1.1$ \\
\hline $\mathrm{MC}$ & I & $7.6 \pm 1.4 \#$ & $11.4 \pm 2.0 \#$ & $28.3 \pm 8.4 \#$ & $63.1 \pm 3.9 \#$ & $4.9 \pm 1.7 \#$ \\
\hline $\mathrm{H}$-Crocetin & 80 & $5.6 \pm 0.9^{* *}$ & $8.1 \pm 1.8^{* *}$ & $16.6 \pm 3.5^{\star \star}$ & $56.1 \pm 5.9^{* *}$ & $3.0 \pm 0.6^{\star *}$ \\
\hline M-Crocetin & 40 & $6.3 \pm 1.2^{*}$ & $9.0 \pm 1.7^{*}$ & $18.5 \pm 7.5^{*}$ & $57.4 \pm 7.0^{*}$ & $3.2 \pm 0.8^{*}$ \\
\hline L-Crocetin & 20 & $6.6 \pm 1.1$ & $9.3 \pm 2.1^{*}$ & $20.3 \pm 7.0^{*}$ & $60.5 \pm 3.2$ & $3.6 \pm 1.2^{*}$ \\
\hline H-HSYA & 80 & $6.1 \pm 0.8^{*}$ & $8.7 \pm 2.3^{*}$ & $18.5 \pm 4.4^{*}$ & $57.1 \pm 3.6^{\star *}$ & $3.0 \pm 0.7^{* *}$ \\
\hline M-HSYA & 40 & $6.3 \pm 1.3^{*}$ & $8.9 \pm 2.5^{*}$ & $19.8 \pm 7.0^{*}$ & $59.0 \pm 3.3^{*}$ & $3.3 \pm 1.0^{*}$ \\
\hline L-HSYA & 20 & $6.6 \pm 1.4$ & $8.9 \pm 2.5^{\star}$ & $19.9 \pm 7.2^{*}$ & $60.4 \pm 3.9$ & $3.3 \pm 1.0^{*}$ \\
\hline Aspirin & 50 & $6.1 \pm 0.9^{*}$ & $8.2 \pm 1.5^{\star *}$ & $17.3 \pm 5.0^{* *}$ & $59.3 \pm 2.8^{*}$ & $3.0 \pm 0.4^{* *}$ \\
\hline
\end{tabular}

${ }^{*} P<0.05,{ }^{*} p<0.01$ compared with model control group; \#p $<0.05$ compared with normal control group

The data showed that treatment groups had significant effects on TXB2 and 6- keto-PGF1a, and dose-dependent effects were noted in TXB2 measurements. All crocetin dose groups exhibited more significant effects on clotting time compared with HSYA groups, while the L - HSYA group had no significant effect. In PAI, crocetin and HSYA both exhibited significant differences, and no dose-dependent effects were observed. In addition, assessment of PC and PS [13], which cause coagulation and fibrinolytic system disorders when lacking, crocetin had a greater and significant therapeutic effect, especially in PS. However, the L - HSYA group had no effect on PC. Crocetin had significant effects on Ddimer, F1 +2 and especially TAT, with the exception of the effect of $L$ - crocetin on D-dimer. HSYA minimally influenced in F1 +2 and the effects were less than those noted for crocetin. All results are presented in Figures 5 - 6 .

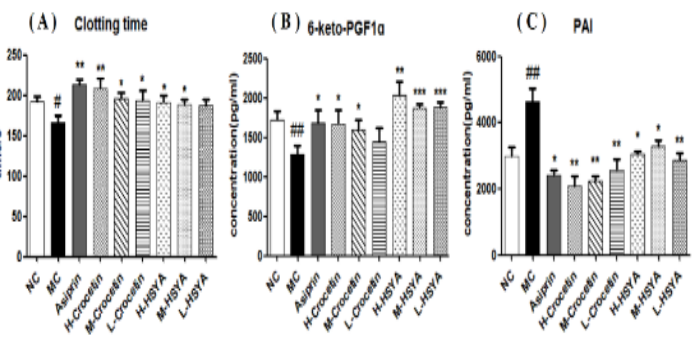

Figure 5: Determination of clotting time (A), 6-ketoPGF1a (B) and PAI (C); ${ }^{*} p<0.05,{ }^{* *} p<0.01,{ }^{* * *} p<$ 0.001 compared with model control group; \#p $<0.05$, \#p $<0.01$ compared with normal control group

\section{DISCUSSION}

The multi-target and multi pathway characteristics of network pharmacology combined with the advantages of TCM could be useful in the mechanistic analysis of disease treatment. Data analysis revealed the same seven targets in the top ten targets. The results indicated a high degree pathway overlap.
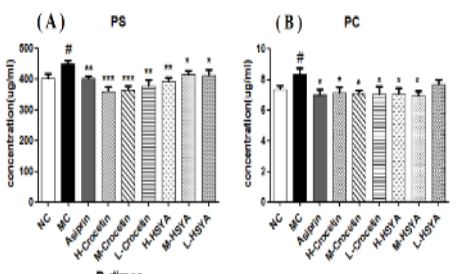

(C) TXB2

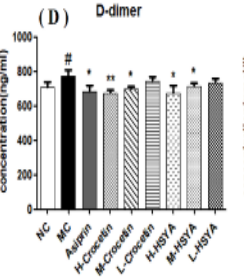

(E) $\quad \mathrm{F} 1+2$

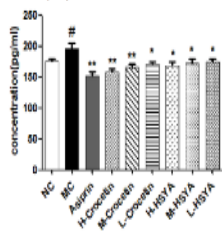

(F) TAT
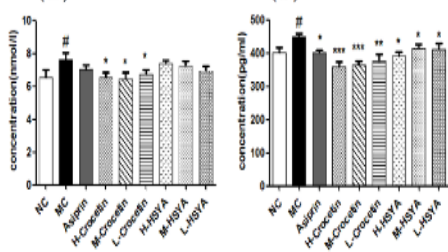

Figure 6: Determination of PS (A), PC (B), TXB2 (C), D-dimer (D), F1 + 2 (E) and TAT (F); ${ }^{*} p<0.05,{ }^{*} p<<$ $0.01,{ }^{* \star *} p<0.001$ compared with model control group ; $\# p<0.05$ compared with normal control group

Eight identical pathways were ranked among the top 10. Therefore, a common mechanism may exist for saffron and HongHua, e.g., blood composition, vascular endothelium, and cell tissue. In addition, in GO enrichment analysis of targets of saffron and Honghua, the top P-value ranked terms among the three terms were also same, reconfirming the functional similarities in the disease pathway.

In the pharmacological validation experiment, adrenaline hydrochloride injection strengthened vasoconstriction in rats. In addition, the body's stress response caused by ice-swimming stimulation accelerated the secretion of endogenous adrenaline, thus aggravating the vasospasm and increasing the internal pressure. These effects ultimately caused endothelial injury in rats and induced coagulation and fibrinolysis system disorders in rats. A previous study reported that a hypercoagulable state is equivalent to a prethrombotic state that promotes blood coagulation and thrombosis, and thrombin is regarded as a key coagulation factor in the prethrombotic state. With a short half-life, 
thrombin is easily covalently bound to TAT by antithrombin under physiological conditions $[14,15]$. F1 + 2 [16], a polypeptide fragment generated after prothrombin activation, is a sensitive molecular marker of early coagulation activation in the body and often be used as one of the early diagnostic indicators for thrombosis.

The fibrinolytic system is important in the maintenance of normal permeability of the vessel wall, blood flow state and tissue repair and can achieve therapeutic effects by dissolving thrombus [17]. PAI [18] participates in the fibrinolytic system reaction and acts indirectly on the dissolution of thrombus; while D-dimer [19] is a specific degradation product produced by the activation and hydrolysis of fibrin, and increased D-dimer production affects intravascular thrombosis. In addition, 6-keto-PGF1a [20,21] is a metabolite of prostacyclin, which is produced by vascular endothelial cells, and exhibits strong inhibitory effects on platelet aggregation and vasoconstriction.

In this research, all HSYA groups had no effect on $\mathrm{F} 1+2$ and exhibited a weaker effect on TAT than crocetin. These finding indicate that HSYA played a weak role in thrombin-involved antithrombotic events. However, HSYA groups exhibited very significant effects on 6-ketoPGF1a $(p<0.01, p<0.001)$, and these effects were superior to those noted for crocetin groups. These results indicate that HSYA may have a greater influence on the regulation of 6-ketoPGF1a_in antithrombotic effect.

\section{CONCLUSION}

The network pharmacology results show that saffron and Honghua exhibit similar a antithrombotic mechanism. In the pharmacological validation experiment, crocetin and HSYA exhibit significant antithrombotic effects, while crocetin exhibits a stronger effect than HSYA in thrombin regulation. Thus, anticoagulation and platelet activation are the primary therapeutic mechanisms of saffron and Honghua. However, we cannot ignore the differences in target paths. The interaction with, and joint development of saffron and Honghua deserves further study, and would likely yield practical therapeutic results of great value.

\section{DECLARATIONS}

\section{Acknowledgement}

We thank the National Natural Science Foundation of China (no. 81703688), Construction of provincial and ministerial level scientific research platform in 2018 - natural product development and utilization of international science and technology cooperation base, The development in key technology for deep processing of Chinese medicinal herbs (saffron) in Zhejiang province and the series of functional foods (no. 2015C02032) and China Iraq Saffron International Joint Research Center (2017C04010) for research support. I want to show my sincere gratitude to my supervisor, Ping Wang, for her instructive advice and useful suggestions in this research. I thank my lab mates for their assistance with the experiments.

\section{Conflict of interest}

No conflict of interest is associated with this work.

\section{Contribution of authors}

We state that this work was performed by the authors named in this article and that all liabilities pertaining to claims relating to the content of this article will be borne by authors. In addition, the authors who conceived and designed the study were Ping Wang and Yingpeng Tong. Jinyan Jiang was responsible for the methodology and manuscript writing, Data collection and analysis were completed by Susu Lin, Qiaoqiao Li, and Shanshan Jiang. Yingjie $\mathrm{Hu}$ and Ling Liu participated in the experiments. Yu Jiang and Shuting Chen performed the network pharmacology analysis.

\section{Open Access}

This is an Open Access article that uses a funding model which does not charge readers or their institutions for access and distributed under the terms of the Creative Commons Attribution License (http://creativecommons.org/licenses/by/ 4.0) and the Budapest Open Access Initiative (http://www.budapestopenaccessinitiative.org/rea d), which permit unrestricted use, distribution, and reproduction in any medium, provided the original work is properly credited.

\section{REFERENCES}

1. Kyriakoudi A, Tsimidou MZ. Latest advances in the extraction and determination of saffron apocarotenoids. Electrophoresis 2018; 39(15): 1846-1859.

2. Zhou XD, Tang $L Y, X u Y L$, Zhou GH, Wang ZJ. Towards a better understanding of medicinal uses of Carthamus tinctorius $L$. in Traditional Chinese Medicine: A phytochemical and pharmacological review. J Ethnopharmacol 2014; 151(1): 27-43.

Trop J Pharm Res, July 2020; 19(7): 1447 
3. Liu JL, Mu JX, Zheng $C L$, Chen $X T$, Guo ZH, Huang $C$, Fu YX, Tian GH, Shang HC, Wang YH. SystemsPharmacology Dissection of Traditional Chinese Medicine Compound Saffron Formula Reveals Multiscale Treatment Strategy for Cardiovascular Diseases. Sci Rep 2016; 6: 19809.

4. Li HX, Han SY, Wang XW, Ma X, Zhang K, Wang L, Ma $Z Z$, Tu PF. Effect of the carthamins yellow from Carthamus tinctorius $L$. on hemorheological disorders of blood stasis in rats. Food Chem Toxicol 2009; 47(8): 1797-1802.

5. Christodoulou E, Kadoglou NPE, Kostomitsopoulos N, Valsami G. Saffron: a natural product with potential pharmaceutical applications. J Pharm Pharmacol 2015; 67(12): 1634-1649.

6. Grover SP, Mackman N. Tissue Factor: An Essential Mediator of Hemostasis and Trigger of Thrombosis. Arterioscler Thromb Vasc Biol 2018; 38(4): 709-725.

7. Furie $B$, Furie $B C$. Mechanisms of thrombus formation. $N$ Engl J Med 2008; 359(9): 938-949.

8. Shi $D Z, X u H$, Yin $H J$, Zhang JC, Chen $K Y$. Combination and transformation of toxin and blood stasis in etiopathogenesis of thrombotic cerebrocardiovascular diseases. Zhong Xi Yi Jie He Xue Bao 2008; 6(11): 1105-1108.

9. Council NR. Guide for the Care and Use of Laboratory Animals: National Academies Press; 2010.

10. Chen Q. Pharmacological experimental method of Chinese medicine. Peo Hea Pub Hou, 1994.

11. Long HP, Lin $X Y$, Wang $Y H$, Ren WQ, Shao L, Zhang W, Tan YS. Explore mechanism of Compound Uncaria Hypotensive Tablet for hypertension based on network pharmacology. Zhongguo Zhong Yao Za Zhi 2018; 43(7): 1360-1365.

12. Zong Y, Dong HL, Chen T, Yan S, Jiang GR. Study on the mechanism of Baicalin-Coptis on the treatment of type 2 diabetes mellitus based on network pharmacology. Chin J Integr Med 2019; 50(4): 888-894.

13. Reznik SE, Fricker LD. Carboxypeptidases from $A$ to $Z$ : implications in embryonic development and Wnt binding. Cell Mol Life Sci 2001; 58(12-13): 1790-1804.

14. Al DR, De LB, Hemker HC. Thrombin generation: What have we learned? Blood Rev 2012; 26(5): 197-203.

15. Ten CH, Hemker HC. Thrombin Generation and Atherothrombosis: What Does the Evidence Indicate? J Am Heart Assoc 2016; 5(8): e003553.

16. Borgen $P O$, Reikeras $O$. Prothrombin fragment $F 1+2$ in plasma and urine during total hip arthroplasty. J Orthopaedics 2017; 14(4): 475-479.

17. Huang YS, Koo M, Chen JC. The association between tinnitus and the risk of ischemic Cerebrovascular disease in young and middle-aged patients: A secondary case-control analysis of a nationwide, population-based health claims database. PLOS One, 2017; 12 (11): e0187474.

18. Li H, Zhang B, LU S, Ji DG, Ding $M$, Ye YS, Sun DJ. SiRNA - mediated silencing of, PAl-1, gene acts as a promoter over the recalizatinaon of endothelial progenitor cells in rats with venous thrombosis. J Cell Physiol 2019; 234(11): 19921-19932.

19. Ding $M$, Zhang $Q Y$, Jing $Y$, Chen $X H$, Geng $X L$, Xie SJ, Zhang $X Q$, Yang $Z$. Application of combined detection of $D$-dimer and fibrinogen in diagnosis of deep venous thrombosis of lower extremities. J Third Mili Med Uni 2016, 38(16): 1885-1888.

20. Zhang JR. Physiology (4 edition). Peo Hea Press 1996; 76.

21. He $W H$, Wang $Z Q$, Jiang $M L$, Jiang $X H$, Zhong $D$. Experimental study on the hemostatic mechanism of Jiao Al Decoction. W J Trad Chi West Med 2012, 7(12): 1032-1034. 